\title{
A Beam Finite Element Model for Efficient Analysis of Wire Strands
}

\author{
Chunlei $\mathrm{Yu}^{1}$, Wenguang Jiang ${ }^{1, *}$, Cai Liu ${ }^{1}$, Jianying Cui ${ }^{2}$ \\ ${ }^{1}$ School of Mechanical Engineering, Yanshan University, Qinhuangdao 066004, Hebei, China \\ ${ }^{2}$ Juli Sling Co. Ltd., Baoding 072550, Hebei, China
}

\begin{abstract}
A beam finite element model (FEM) for efficient analysis of the mechanical behavior of wire strands is presented. Two-noded elasticplastic beam elements were used for wire discretization. Hertz contact theory was implemented via newly developed node-to-node compression-only contact element to simulate the wire-to-wire contacts. The first numerical example demonstrated is the analysis of a three layered 19-wire strand under axial tensile load. The results showed excellent agreement with those obtained from the accurate full three-dimensional solid FEM of Jiang et al. The degrees of freedom for the beam FEM is only about $4 \%$ of the solid FEM. The second verification example presented is the simulation of a six-layered 91-wire strand under axial tensile load. For the global behavior of the strand, the finite element results showed better agreement with the experimental data than Costello's elasticity theory. For this multilayered strand, it could be extremely hard to implement an accurate full three-dimensional solid FEM.
\end{abstract}

Keywords: Wire strand, Finite element model, Beam element, Hertz contact, Tensile load

(Received on July 3, 2016; Revised on November 28, 2016; Accepted on April 8, 2017)

(C) 2017 Totem Publisher, Inc. All rights reserved.

\section{Introduction}

Wire strands are a wide class of useful engineering components and play an indispensable role in various engineering applications such as suspension bridges, sports stadia and cable-membrane structures, etc. The major advantage of the wire strands is their capacity to support large axial loads with comparatively small bending or torsional stiffness.

In the last several decades, considerable progress has been made in the development of theories and models to study the mechanical behavior of wire strands. Literature survey shows that analytical models are commonly applied to relatively simple cases in which approximations and assumptions have been made [1-5]. The effects of more complicated factors such as contact deformation, wire uneven bending due to discontinuous contacts and material plasticity, etc., are very difficult to predict analytically. With the development of the finite element (FE) method and the associated computing capacity, it is possible now to analyze more complex strand behavior including the actual geometry and other factors of influence [6]. Many threedimensional solid finite element models (FEMs), which can take into account all the influence factors noted above and provide detailed information about the strand mechanical behavior, have been presented. However, a majority of the published solid FEMs are limited to the relatively simple wire strand constructions with only one or two helical wire layers [7-10]. For multilayered wire strands, the behavior simulation using three-dimensional solid model can be very expensive and the desired degree of refinement is frequently not within the practical limits of computer resources due to the strand complex geometry and the high nonlinearity of the numerous inter-wire contacts involved. Therefore, there is a requirement for an efficient FEM, which can consider the influence factors as much as possible.

In this paper a beam FEM for wire strands has been developed. The major influencing factors such as contact deformation, wire diameter contractions due to Poisson's ratio effect, wire uneven bending effects and material plasticity have all been considered. The strand wires have been discretized using traditional two-noded beam elements. Inter-wire contacts are assumed to obey the Hertz contact theory. The simulation of these contacts has been realized by using newly developed nodeto-node contact elements located at all possible contact positions. Numerical examples have been given to show the accuracy and efficiency of the beam FEM developed.

\footnotetext{
* Corresponding author.

E-mail address: wgj@ysu.edu.cn
} 


\section{Finite element model}

A typical geometry of the wire strand analyzed in this paper is shown in Fig. 1. In general, it comprises a straight core wire surrounded helically by several successive layers of round section wires. The core wire is defined as the first-layer wire. For an arbitrary wire in the layer $i(i=1,2, \ldots, n)$, the radius of the wire is $R_{i}$ and the helical angle is $\alpha_{i}$, where $n$ is the total number of wire layers in the strand. The helical directions for adjacent layers of the helical wires are opposite, which results in point contact between these layers. The contacts between the core wire and each of the second-layer wires are line contacts [6]. The strand is loaded axially with an axial force, $F$, and an axial twist moment, $M$, which represents the most basic loading case.

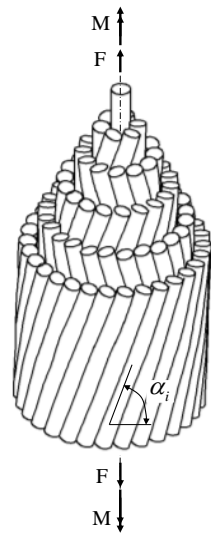

Fig.1: A typical geometry of wire strand

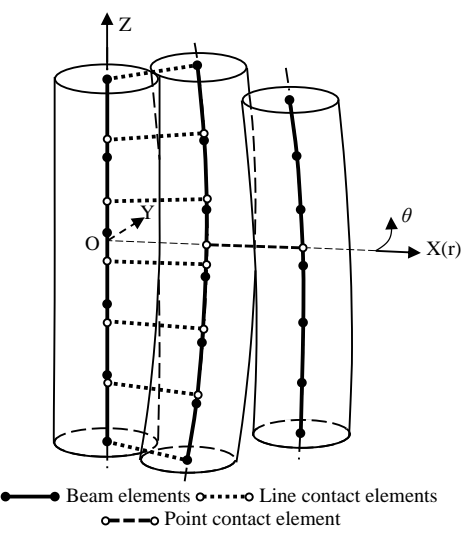

Fig.2: Representative part of strand geometry and beam FEM discretization

Fig. 2 shows a small typical representative part of the wire strand geometry with a core wire helically surrounded by a second-layer wire and a third-layer wire, which has point contact with the second-layer wire. This figure also shows a sample FE discretization of the strand wires using beam elements, which are established along the wire centerlines. The beam elements used are the two-noded elastic-plastic elements, having six degrees of freedom on each node, i.e. translations in $x, y$ and $z$ directions and rotations about the $x, y, z$ directions. These elements are based on Timoshenko beam theory and have capabilities to simulate the combined effects of tension, shear, torsion and bending. The newly developed node-to-node Hertz contact elements established at all possible contact positions between wires are also shown in Fig. 2. There are two types of contact elements, i.e. the line contact element and point contact element. The densely distributed contact elements connecting the centerlines of the core wire and the centerlines of the second-layer wires are used to simulate the continuous line contacts between these two layers of wires, also known as line contact elements. The contact element located at the shortest distance between the helical centerline of the second-layer wire and the helical centerline of the third-layer wire is used to simulate the point contact between these two successive layers of wires, which is also called point contact element.

Using the same rule of the FE discretization for the strand representative part discussed above, the beam FEM of the whole strand can be constructed. The contact positions for the Hertz contact elements establishment can be determined from the strand geometry. For line contact, the contacts locate on the wire contact lines. For point contact, the contact positions can be determined using the equations of contact lines and the axial space between two adjacent contact points located on the same contact line [7].



Fig. 3: Example $P-\delta$ relationship for line contact

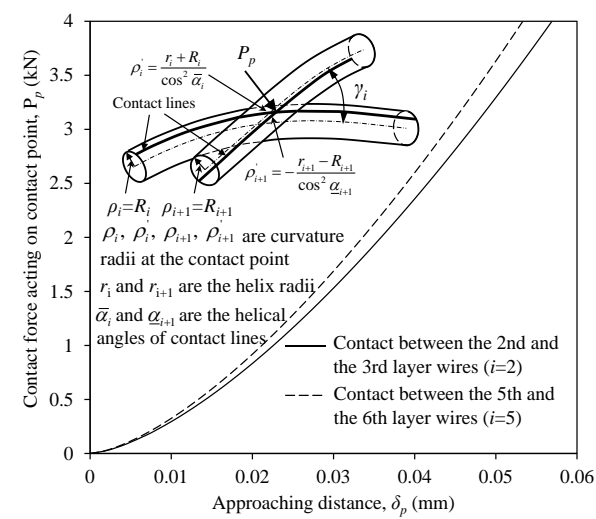

Fig. 4: Example $P-\delta$ relationships for point contact 


\subsection{Development of Hertz contact elements}

As the large number of distributed wire-to-wire contacts inherent within the wire strand have significant influences on the strand mechanical behavior, these contacts have to be considered in detail in a rational simulation model. In this paper, the node-to-node compression-only contact element has been developed to fulfil this task. The new contact element is defined by two nodes having three degrees of freedom on each, i.e. translations in $x, y$ and $z$ directions, and its $x$-axis is defined from one end node to the other. This element can only transfer axial compression force along its $x$-axis direction. Provided that the significant dimensions of the contact area are small compared with the curvature radii of the contacting wires, the Hertz contact theory could be applied to the wire-to-wire contact analyses with sufficient accuracy. The analytical Hertz contact relationship between the contact force, $P$, and the approaching distance between the centerlines of the two contacting wires at the contact location, $\delta$, has been used to define the contact element behavior, and these equations are given referring to literature [2,6]. Fig. 3 gives an example relationship of $P_{l}-\delta_{l}$ for the line contact and Fig. 4 shows example $P_{p^{-}} \delta_{p}$ curves for point contacts. These example curves are achieved from the second verification example, which is illustrated in the Section 3.2 of this paper. As the relationship between $P$ and $\delta$ is essentially nonlinear, an iterative solution procedure has to be used. In this paper, a secant modulus method has been employed to upgrade the stiffness of the contact elements for the iterative nonlinear solution procedure.

\subsection{Incorporating Poisson's ratio effect}

As strand wires are mainly subjected to high tension load, wire diameter will contract. This is known as the Poisson's ratio effect. The wire diameter contraction due to the Poisson's ratio effect has been considered as an additional part to be incorporated into the approaching distance of the centerlines of the contacting wires. For a contact element between the layers $i$ and $i+1(i=1,2, \ldots, n)$, the approaching distance caused by Poisson's ratio can be calculated using

$$
\delta_{\mu}^{i}=-\left(\mu \varepsilon_{i}^{e}+\mu_{p} \varepsilon_{i}^{p}\right) R_{i}-\left(\mu \varepsilon_{i+1}^{e}+\mu_{p} \varepsilon_{i+1}^{p}\right) R_{i+1}
$$

where $\mu$ is the Poisson's ratio in elastic range, $\mu_{p}$ is the Poisson's ratio in the plastic range $\left(\mu_{p}=0.5\right.$ herein for volumetric incompressible). $\varepsilon_{i}^{e}$ and $\varepsilon_{i}^{p}$ denote the elastic and plastic strain components of the axial strain of the $i$ th layer wire $\varepsilon_{i}$, respectively. $\varepsilon_{i+1}^{e}$ and $\varepsilon_{i+1}^{p}$ are the elastic and plastic strain components of $\varepsilon_{i+1}$, respectively.

A bilinear isotropic hardening material model is used, thus $\varepsilon_{i}^{e}, \varepsilon_{i}^{p}, \varepsilon_{i+1}^{e}$ and $\varepsilon_{i+1}^{p}$ can be determined easily based on if the wire material enters into plastic range. During solution process, the value of $\delta_{\mu}^{i}$ could be updated iteratively at every subloading step.

\subsection{Incorporating Poisson's ratio effect}

In engineering application wire strands are normally well lubricated; thus, the frictions between the wire-to-wire contacts are ignored for simplicity. For the symmetric feature of the strand structure and the axial extension loading case, the slidings between the contacting wires are very small and the inter-wire contacts will occur in the strand radial direction. The contact elements are designed to establish the radial contact relationships, and the possible small free slidings are allowed in the perpendicular directions. In the beam element strand model, the beam elements and the contact elements are established separately, i.e. they have their own separate sets of nodes. Constraint equations are used to build the connections between the nodal degrees of freedom of the contact elements and the nodal degrees of freedom of the beam elements based on their relative positions. For a contact element laid between arbitrary two adjacent layers $i$ and $i+1(i=1,2, \ldots, n)$, one node $\left(\mathrm{N}_{\mathrm{c} 1}\right)$ of the contact element has the same displacement as its coincident point inside the $i$ th layer beam element. The displacement interpolations of this coincident point of the beam element are used to tie the displacements of this node of the contact element, i.e.

$$
u_{r 1}=N_{1} u_{r 1}^{i}+N_{2} u_{r 2}^{i}, u_{\theta 1}=N_{1} u_{\theta 1}^{i}+N_{2} u_{\theta 2}^{i}, u_{z 1}=N_{1} u_{z 1}^{i}+N_{2} u_{z 2}^{i}
$$

where $u_{r 1}, u_{\theta 1}$ and $u_{z 1}$ are the displacement components of the node $\mathrm{N}_{\mathrm{c} 1}$ in the global $r, \theta$ and $z$ directions, $u_{r 1}^{i}, u_{r 2}^{i}, u_{\theta 1}^{i}$, $u_{\theta 2}^{i}, u_{z 1}^{i}$ and $u_{z 2}^{i}$ are the displacement components of the two nodes $\left(\mathrm{N}_{\mathrm{b} 1}^{i}\right.$ and $\left.\mathrm{N}_{\mathrm{b} 2}^{i}\right)$ of the ith layer beam element in the global $r, \theta$ and $z$ directions. The displacement interpolation function $N_{1}(s)=(1-s) / 2$ and $N_{2}(s)=(1+s) / 2$ are defined in the natural coordinate system of the beam element, which has variable $s$ that varies from -1 to +1 . The $s$ determines the coincident point within the beam element and can be calculated using: $s=-1+2 \overline{\mathrm{N}_{\mathrm{cl}} \mathrm{N}_{\mathrm{b} 1}^{i}} / \overline{\mathrm{N}_{\mathrm{b} 1}^{i} \mathrm{~N}_{\mathrm{b} 2}^{i}}$, where the superbars represent the distances between the two nodes.

For the other node $\left(\mathrm{N}_{\mathrm{c} 2}\right)$ of the contact element, it has the same radial displacement as its coincident point inside the 
$(i+1)$ th layer beam element. The radial displacement interpolation for this node of the contact element is

$$
u_{r 2}=N_{1} u_{r 1}^{i+1}+N_{2} u_{r 2}^{i+1}
$$

where $u_{r 2}$ is the radial displacement component of the node $\mathrm{N}_{\mathrm{c} 2}, u_{r 1}^{i+1}$ and $u_{r 2}^{i+1}$ are the radial displacement components of the two nodes $\left(\mathrm{N}_{\mathrm{b} 1}^{i+1}\right.$ and $\mathrm{N}_{\mathrm{b} 2}^{i+1}$ ) of the $(i+1)$ th layer beam element respectively, parameter $s$ to determine the coincident point within the beam element in the (i+1)th layer can be calculated using: $s=-1+2 \overline{\mathrm{N}_{\mathrm{c} 2} \mathrm{~N}_{\mathrm{b} 1}^{i+1}} / \overline{\mathrm{N}_{\mathrm{b} 1}^{i+1} \mathrm{~N}_{\mathrm{b} 2}^{i+1}}$.

To keep the contact element stay aligned with the radial direction of the strand after loading, the node $\left(\mathrm{N}_{\mathrm{c} 2}\right)$ of the contact element is set to have the same angular displacement with the other node $\left(\mathrm{N}_{\mathrm{c} 1}\right)$ in the global $\theta$ direction. For the line contact elements $(i=1)$, it can be realized by using

$$
u_{\theta 2}=N_{1} u_{\theta 1}^{2}+N_{2} u_{\theta 2}^{2}
$$

where $u_{\theta 2}$ is the displacement component of the node $\mathrm{N}_{\mathrm{c} 2}$ in the global $\theta$ direction, $u_{\theta 1}^{2}$ and $u_{\theta 2}^{2}$ are displacement components of the two nodes $\left(\mathrm{N}_{\mathrm{b} 1}^{2}\right.$ and $\mathrm{N}_{\mathrm{b} 2}^{2}$ ) of the second layer beam element in the global $\theta$ direction, respectively.

For the point contact elements $(i=2,3, \ldots, n)$, it can be written as

$$
u_{\theta 2} / r_{i+1}=u_{\theta 1} / r_{i}
$$

where $r_{i}$ and $r_{i+1}$ are helix radii for the $i$ th layer wires and $(i+1)$ th layer wires, respectively.

In the global $z$ direction, the node $\left(\mathrm{N}_{\mathrm{c} 2}\right)$ of the contact element is set to have the same displacement with the node $\left(\mathrm{N}_{\mathrm{c} 1}\right)$ by using

$$
u_{z 2}=u_{z 1}
$$

where $u_{z 2}$ is the displacement component of the node $\mathrm{N}_{\mathrm{c} 2}$ in the global $z$ directions.

For all contact elements, these constraint equations should be established. As these equations are linear equations of the degrees of freedom, they can be easily incorporated into the system of equations during solution process.

\section{Numerical examples}

The beam FEM proposed has been implemented using ANSYS FE software. The analysis of two typical wire strand constructions has been conducted to show the accuracy of the beam FEM.

\subsection{The first numerical example}

This verification example is the analysis of a three layered 19-wire strand. The reason for choosing this relatively simpler strand construction is that accurate full three-dimensional solid FE modeling results could be achieved [7] and these could be used to validate the newly developed beam FEM in detail. Table 1 gives the geometry data of this strand. The diameter of the center wire is larger than the diameters of the helical wires, which ensures that contact occurs only between adjacent layers of wires.

Table 1 The geometric data of the three layered 19-wire strand

\begin{tabular}{cccccc}
\hline $\begin{array}{c}\text { Layer } \\
\text { number } i\end{array}$ & $\begin{array}{c}\text { Number of } \\
\text { wires } m_{i}\end{array}$ & $\begin{array}{c}\text { Wire diameter } \\
2 R_{i}(\mathrm{~mm})\end{array}$ & $\begin{array}{c}\text { Pitch length } \\
p_{i}(\mathrm{~mm})\end{array}$ & $\begin{array}{c}\text { Helical angle } \\
\alpha_{i}\left({ }^{\circ}\right)\end{array}$ & Helical direction \\
\hline 1 & 1 & 5.20 & - & 90 & - \\
2 & 6 & 4.55 & 107.3 & 74.1 & RH \\
3 & 12 & 4.55 & 207.4 & 74.1 & LH \\
\hline
\end{tabular}

Fig. 5 shows the beam FEM for the three layered 19-wire strand analyzed. The length of the strand beam FEM used for analysis, $\Delta z$, is ten times the strand diameter, $d=23.4 \mathrm{~mm}$. The size of the beam element is about 0.3 times the wire radius. The spacing between adjacent line contact elements is $0.2 R_{1}$ along the strand axial direction. The beam FEM of the strand consists of 3966 beam elements and 1650 contact elements. The total number of nodes for both beam elements and contact elements is 6109. The degrees of freedom for this strand model is only about 4\% of the accurate three-dimensional solid FEM [7] which consists of 137928 elements and 139191 nodes. The Von Mises yield criterion was assumed. A bilinear isotropic hardening material model was used. The material properties for the wires are given in Table 2. 


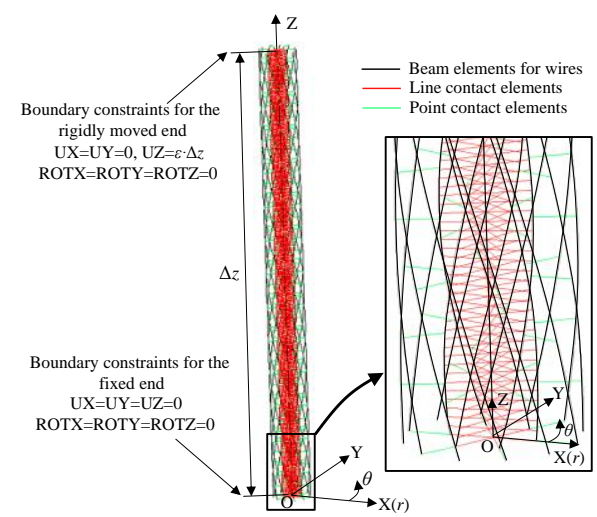

Fig. 5: The beam finite element mesh and boundary conditions for the 19-wire strand

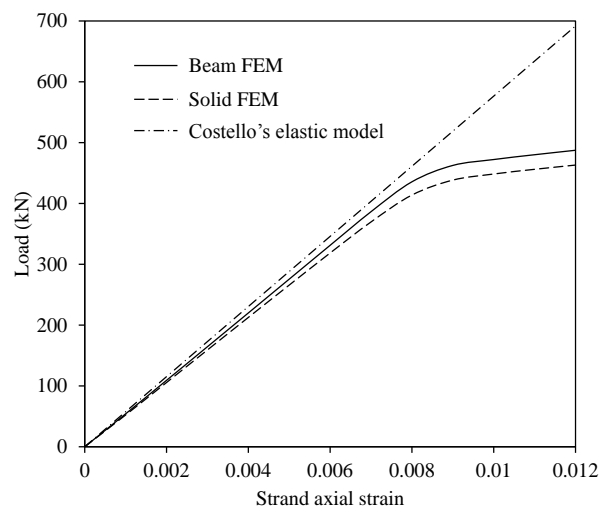

Fig. 6: Variation of axial load with strand axial strain for the 19-wire strand

The strand was subjected to non-rotation tension at the two ends of the model (Fig. 5). One simple way of implementing this is to fully constrain one end cross-section of the strand model and rigidly move the other end cross-section of the strand model away along the strand axial direction. In this analysis, a mean strand axial strain, $\varepsilon$, of 0.012 was applied in increments of 0.001. The FE analysis results from the beam FEM have been compared in detail with those from the accurate threedimensional solid FEM [7]. The Costello's elasticity model [1] has also been given in the comparison where applicable.

Table 2 Material properties for wires

\begin{tabular}{ccccc}
\hline Young's modulus & Plastic modulus & Yield stress & Limit stress & Poisson's ratio \\
$E(\mathrm{GPa})$ & $E_{p}(\mathrm{GPa})$ & $\sigma_{s}(\mathrm{GPa})$ & $\sigma_{b}(\mathrm{GPa})$ & $\mu$ \\
\hline 210 & 24.6 & 1.54 & 1.80 & 0.3 \\
\hline
\end{tabular}

Fig. 6 shows the strand axial load as a function of the applied mean strand axial strain. Due to the consideration of contact deformation, the beam FEM prediction is closer to the result obtained from the accurate solid FEM than the Costello's elastic theory. The slightly higher tensile rigidity prediction from the beam FEM is mainly due to that the beam FEM has not considered the loss of axial load-bearing ability of the local material in the vicinity of the contact regions. This phenomenon had been discussed in detail in reference [6].

The variation of the contact forces acting on sequential contact points along a third-layer wire is given in Fig. 7. It can be seen that within the transition regions (a length of about $1.5 \mathrm{~d}$ from both fixed ends of the strand), the contact forces show obvious variation. Within the middle region free from termination effect, the contact forces at all crossing contacting points are nearly the same. Due to the implementation of accurate helically symmetric and cyclic symmetric boundary conditions, the results from the comparison solid FEM are the results free from the termination effects [7]. From this figure it can be seen that the results predicted by the beam FEM are in good agreement with those obtained from the accurate solid FEM.

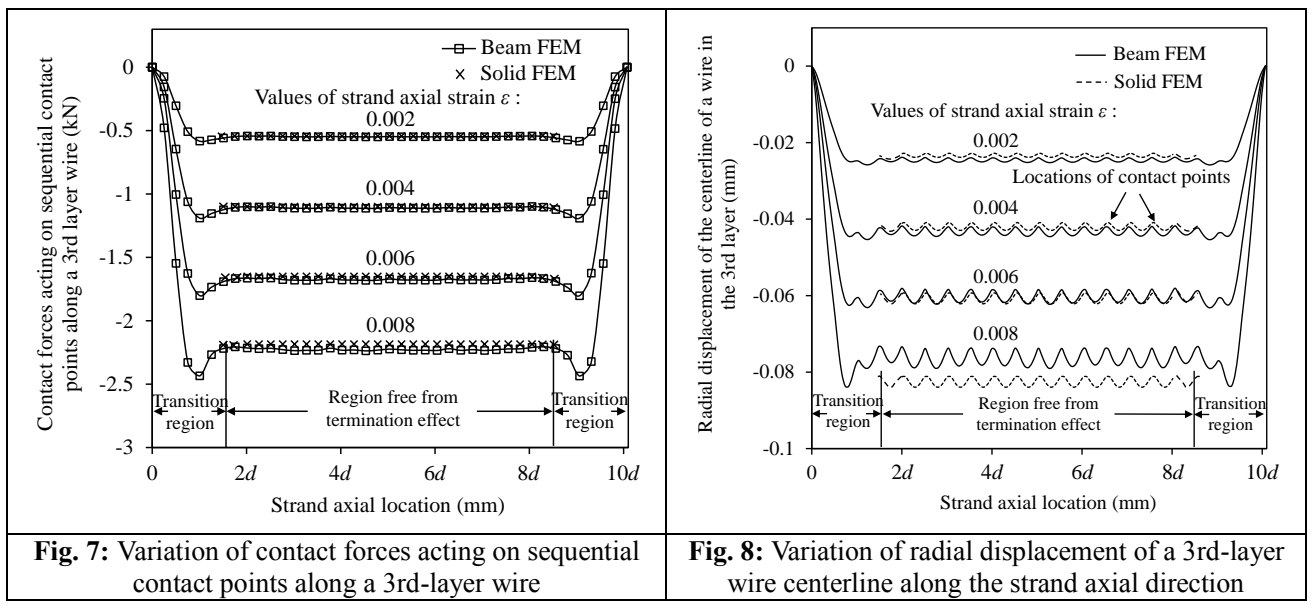

The variation of the radial displacements of the centerline of a third-layer wire along the strand axial direction is given in Fig. 8. The prediction results from the present beam FEM show very good agreement with those from the solid FEM for low 
extension strains $(\varepsilon \leq 0.006)$. However, when strand axial strain reaches 0.008 , the prediction of the radial displacement from the beam FEM is smaller than that from the solid FEM. The main reason for this is that at this load level, the bulk of strand material has experienced plastic yielding, and the elastic Hertz contact theory is no longer valid in such case. These wave-like radial displacement patterns indicate that these helical wires are subjected to uneven bending due to the discontinuous point contact.

\subsection{The first numerical example}

The second numerical example is the simulation of a six layered 91-wire strand shown in Fig. 1. Table 3 gives the geometry data of this strand. The inter-wire contacts occur only between the adjacent layers of wires. The length of the strand model used is ten times the strand diameter. The same mesh density, wire material properties and loading case are the same as the first numerical example. The beam FEM is composed of 49811 beam elements and 7344 contact elements. The total number of nodes is 62015. A mean strand axial strain of 0.012 was applied in increments of 0.001 in this analysis.

Table 3 The geometric data of the six layered 91-wire strand

\begin{tabular}{cccccc}
\hline $\begin{array}{c}\text { Layer } \\
\text { number } i\end{array}$ & $\begin{array}{c}\text { Number of } \\
\text { wires } m_{i}\end{array}$ & $\begin{array}{c}\text { Wire diameter } \\
2 R_{i}(\mathrm{~mm})\end{array}$ & $\begin{array}{c}\text { Pitch length } \\
p_{i}(\mathrm{~mm})\end{array}$ & $\begin{array}{c}\text { Helical angle } \\
\alpha_{i}\left({ }^{\circ}\right)\end{array}$ & Helical direction \\
\hline 1 & 1 & 5.20 & - & 90 & - \\
2 & 6 & 4.55 & 107.3 & 74.1 & $\mathrm{RH}$ \\
3 & 12 & 4.55 & 207.4 & 74.1 & $\mathrm{LH}$ \\
4 & 18 & 4.55 & 307.5 & 74.1 & $\mathrm{RH}$ \\
5 & 24 & 4.55 & 407.6 & 74.1 & $\mathrm{LH}$ \\
6 & 30 & 4.55 & 507.7 & 74.1 & $\mathrm{RH}$ \\
\hline
\end{tabular}

Fig. 9(a) shows the distribution of axial displacement, $u_{z}$, and Fig. 9(b) shows the distribution of radial displacement, $u_{r}$, at strand mean axial extension strain of 0.004. It can be seen from Fig. 9 that there are non-uniform transition regions close to both ends of the strand. The length of the termination effect influence regions is about $1.5 d$, where $d=50.7 \mathrm{~mm}$ is the nominal strand diameter. The radial contraction of the cross-sections increases as the distance from the ends increases in the two transition regions at both ends. In the middle part, the deformations are quite uniform.

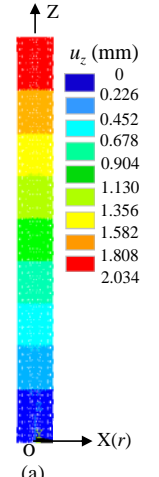

Fig. 9: Contour plot of displacement at a strand axial strain of 0.004: (a) axial displacement, $u_{z}$, and (b) radial displacement, $u_{r}$

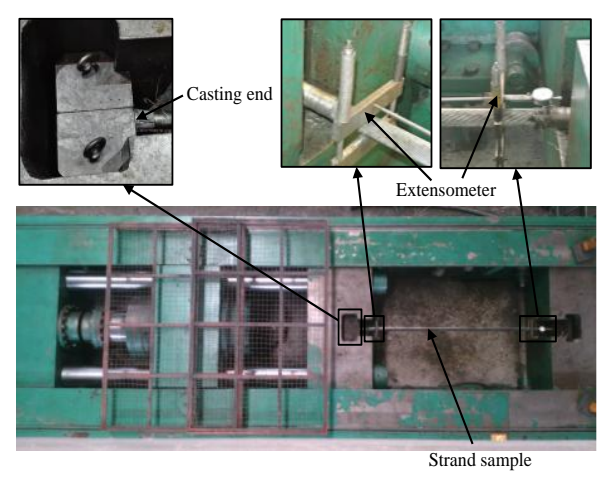

Fig. 10: Tensile experiment for the 91-wire strand

Due to the complex geometry of this 91-wire multilayered strand and considering the many distributed local contacts involved in this strand construction, the implementation of an accurate three-dimensional solid FEM will be extremely difficult if not impossible. Instead, experimental tests of the tensile behavior had been conducted for validation purpose. Fig. 10 shows the arrangement of the tensile experiment. A $5000 \mathrm{kN}$ hydraulic tension test machine was used. The length of the sample strand is $3000 \mathrm{~mm}$ and its ends are processed using the method of casting with zinc alloy. In the implementation of the tensile test, the sample strand was fixed on the tension machine via open-type clamps with conical cavity. Extensometer was used for the measurement of strand elongations and it was mounted on the sample strand with an original length of $1953 \mathrm{~mm}$. Vernier caliper with wide mouth pliers was used to measure the strand diameter. Fig. 11 shows the strand axial load as a function of the applied mean axial strain. It can be seen that the present beam FEM is in good agreement with the experimental data than Costello's elastic model. When the strand axial strain reaches approximately 0.008 , the bulk of the strand material begins to yield. For safety reason, the tensile data had not been collected after the loading level has exceeded $0.6 F_{0}$, where $F_{0}=2400 \mathrm{kN}$ is the experimentally measured breaking load of the strand.

To further investigate the significance of considering the contact deformation in the numerical model, the amount of 
diameter contraction of the strand during the loading process was also experimentally measured. Fig. 12 compares the reductions of the strand diameter at different load levels. It can be seen that the prediction result from the beam FEM compares reasonably well with the experimental values, whereas Costello's elastic model gives too lower predictions for a given mean axial strain. This indicates that the contact deformation should be considered when developing accurate numerical models for multi-layered strands.

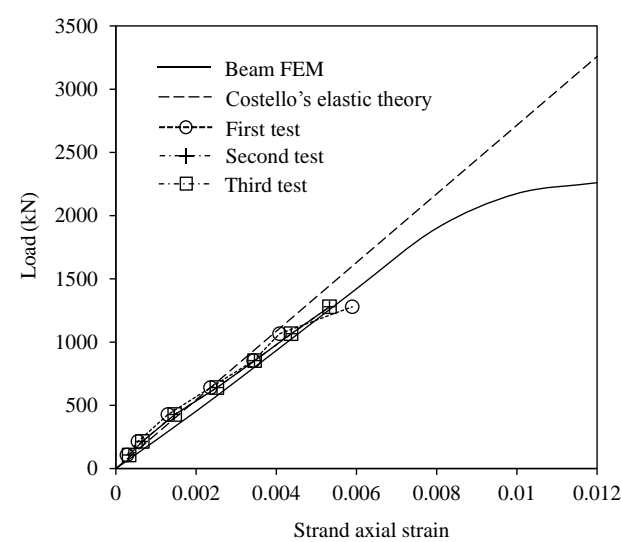

Fig. 11: Variation of axial load with strand axial strain for the 91-wire strand

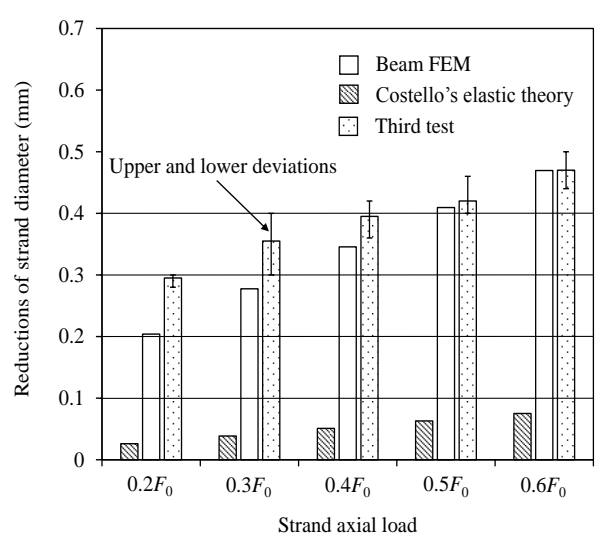

Fig. 12: Comparisons of strand diameter reductions under different loading levels

\section{Conclusions}

An efficient beam FEM has been developed to predict the mechanical behavior of complex wire strands. The wires in the strand were discretized using the traditional two-noded elastic-plastic beam elements. Contacts between strand wires were simulated using the newly developed two-noded contact elements based on the analytical solution of Hertz contact theory. The analysis results of two typical wire strand constructions showed good agreements with those from both the accurate threedimensional solid FEM and the experiments. Compared with solid FEMs, the new beam FEM has much smaller model size. Thus, the methodology could be further extended to build efficient models for wire strands/ropes with complex cross-sections.

\section{Acknowledgement}

This work is supported by Hebei Provincial Natural Science Foundation of China (grant number E2015203196).

\section{References}

1. Velinsky, S. A., Anderson, G. L., Costello, G. A. Wire Rope with Complex Cross Sections. Journal of Engineering Mechanics. 1984; 110(3): 380-391.

2. LeClair, R. A. Axial response of multilayered strands with compliant layers. Journal of Engineering Mechanics. 1991; 117 (12): $2884-2902$.

3. Gnanavel, B. K., Parthasarathy, N. S. Effect of interfacial contact forces in radial contact wire strand. Archive of Applied Mechanics. 2011; 81 (3): 303-317.

4. Argatov, I. Response of a wire rope strand to axial and torsional loads: Asymptotic modeling of the effect of interwire contact deformations International Journal of Solids \& Structures. 2011; 48 (10): 1413-1423.

5. Meng, F. M., Chen, Y. P., Du, M. G., Gong, X. S. Study on effect of inter-wire contact on mechanical performance of wire rope strand based on semi-analytical method. International Journal of Mechanical Sciences. 2016; 115: 416-427.

6. Jiang, W. G., Yao, M. S., Walton, J. M. A concise finite element model for simple straight wire rope strand. International Journal of Mechanical Sciences. 1999; 41 (2): 143-161.

7. Jiang, W. G., Henshall, J. L., Walton, J. M. A concise finite element model for three-layered straight wire rope strand. International Journal of Mechanical Sciences. 2000; 42 (2): 63-86.

8. Judge, R., Yang, Z., Jones, S. W., Beattie, G. Full 3D finite element modeling of spiral strand cables. Construction \& Building Materials. 2012 35: 452-459.

9. Yu, Y. J., Chen, Z. H., Liu, H. B., Wang, X. D. Finite element study of behavior and interface force conditions of seven-wire strand under axial and lateral loading. Constructions \& Building Materials. 2014; 66 (1): 10-18.

10. Chen, Y. P., Meng, F. M., Gong, X. S. Parametric modelling and comparative finite element analysis of spiral triangular strand and simple straight strand. Advances in Engineering Software. 2015; 90: 63-75. 


\section{Biographies}

Mr. Chunlei Yu is a PhD candidate at Yanshan University, China. His major research interests include mechanical behavior of wire rope strand and construction machinery. He has published many papers about his research area.

Prof. Wenguang Jiang is a researcher at Yanshan University, China. His major research interests include mechanical simulation of wire rope and construction machinery. He is invited as a reviewer by the editors of some international journals, such as International Journal of Solids and Structures, International Journal of Mechanical Sciences etc. He has published many papers in related journals.

Prof. Cai Liu is a researcher at Yanshan University, China. His major research interests is computer simulation of plastic forming process. He has published many papers about his research area.

Mr. Jianying Cui is a senior engineer at Juli Sling Co, Ltd., China. His major research interests include the design and manufacture of wire rope and wire rope sling. 